\title{
Disseminated Histoplasmosis in a 13-year-old girl: a case report
}

\author{
*Ubesie AC, Okafo OC, Ibeziako NS, Onukwuli VO, Mbanefo NR, Uzoigwe JC, Bede C, Ibe BC
}

University of Nigeria Teaching Hospital, Ituku/Ozalla, Enugu, Nigeria

\begin{abstract}
Background: Disseminated histoplasmosis is a rare fungal infection and most documented cases are in immunecompromised individuals such as those with acquired immuno-deficiency syndrome.

Objective: To describe a case of disseminated histoplasmosis in an adolescent girl.

Method: We report a case of disseminated histoplasmosis in a 13-year-old adolescent girl. She was admitted for 16 days because of neck masses of 3 years duration, generalized body swelling of 3 months and reduction in urinary output of 2 months. She tested negative for human immunodeficiency virus antibodies.

Result: An autopsy was performed because a definitive diagnosis could not be made while the patient was still alive. The autopsy revealed central caseating areas in the lymph nodes and membranoproliferative glomerulonephritis. The periodic acid-Schiff staining technique for tissues showed viable yeast cells suggestive of histoplasmosis. Zeihl-Neelsen's staining for mycobacteria tuberculosis was negative.

Conclusion: Undiagnosed case of disseminated histoplasmosis while the patient was alive is being reported in a 13-yearold girl. Disseminated histoplasmosis should be considered as a differential diagnosis of childhood chronic infections and malignancies as in Nigeria.
\end{abstract}

Key words: Disseminated histoplasmosis, immune-competent, adolescent girl, Enugu

African Health Sciences 2013; 13(2): 518 - 521http://dx.doi.org/10.4314/ahs.v13i2.45

\section{Introduction}

The first reported case of histoplasmosis in a human, was by Samuel Darling Panama ${ }^{1}$. Histoplasmosis is caused by a fungal infection, Histoplasma capsulatum. There are two varieties of the pathogen, Histoplasma capsulatum var. capsulatum and Histoplasma capsulatum var. duboisii that cause disease in humans ${ }^{2}$

The disease is classified into acute pulmonary histoplasmosis (normal host), disseminated histoplasmosis (immune defect) and chronic pulmonary histoplasmosis (structural defect) ${ }^{3,4,5}$. The acute histoplasmosis is usually asymptomatic, occasionally symptomatic but complications are rare ${ }^{3}$. The disseminated form of the disease usually occurs in immunocompromised individuals such as those with acquired immune-deficiency syndrome (AIDS), transplant recipients, those with haematological malignancies, infants and those on corticosteroids, and it is caused by defective cell mediated immunity in these individuals ${ }^{6}$. It is becoming a common opportunistic infection among individuals with AIDS living in endemic areas ${ }^{7,8,9}$. On the other hand, chronic progressive disseminated histoplasmosis is a term

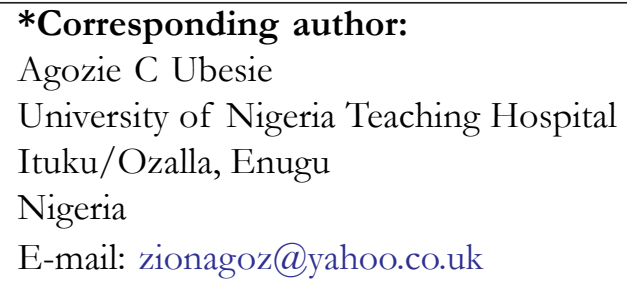

used to describe the slowly progressive and generally fatal infection due to $H$. capsulatum that occurs mostly in older adults who are not overtly immunosuppressed.

The time course of the infection in these patients is measured in months, and the disease is uniformly fatal if not treated ${ }^{4}$. We report a case of disseminated histoplasmosis in a 13-year-old girl that mimicked a number of clinical conditions. We were unable to correctly diagnose the patient while she was alive, necessitating an autopsy.

\section{Case history}

A 13-year-old female was admitted for 16 days and presented with neck masses of 3 years duration, generalized body swelling of 3 months and had a reduction in urinary output for 2 months. The patient took native medications over a period of approximately 1 year in the course of the illness and had two episodes of generalized tonic convulsions 2 months prior to presentation. There was no history of long term steroid use locally or systemically or use of chemotherapy. There was no history of receiving antibiotics for a prolonged period. There was no history of a similar illness in the family or contact with persons with chronic cough.

A physical examination revealed that the patient was in respiratory distress, she was pale, and had generalized body swelling and significant generalized 
lymphadenopathy. She had an elevated blood pressure of $160 / 100 \mathrm{mmHg}$, and her oxygen saturation was less than $88 \%$. The abdomen was grossly distended and it was difficult to delineate any enlarged organ because of massive ascites.

The clinical diagnosis on admission was nephritic syndrome possibly secondary to a lymphoma. Other possibilities considered were human immunodeficiency virus (HIV)-associated nephropathy and disseminated tuberculosis.

HIV antibody screening and a Mantoux test were negative. Haemoglobin was $6 \mathrm{~g} / \mathrm{dl}$ and the erythrocyte sedimentation rate was $95 \mathrm{~mm}$ in the first hour (western green). Patient was not specifically screened for diabetes mellitus or visceral leshmaniasis. Urinalysis however, did not reveal glycosuria but showed significant proteinuria and heamturia. The serum creatinine and urea were elevated with reduced bicarbonate. Total serum protein and albumin were within normal limits. Chest X-ray showed cardiomegaly with pericardial effusion and right sided pleural effusion.

Abdominal ultrasound showed bilateral nephritis. A scheduled biopsy for the neck mass was not performed because the patient was unfit for surgery, but ascitic fluid cytology was negative for malignant cells.

The patient's management involved blood pressure and oedema control, which became refractory. On the $16^{\text {th }}$ day of admission, she had seizures and stopped breathing shortly afterwards. Consent for a post mortem examination was obtained and this was carried out.

An autopsy revealed marked enlargement of the axillary, cervical, hilar, mediastinal, para-aortic, and mesenteric lymph node groups. Microscopic examination of these lymph nodes showed intracellular and extracellular haematoxylinophilic bodies, each surrounded by a small halo as indicated in figure 1.

They measured between 2 and 5 microns in diameter. The characteristic morphological features of Histoplasma were observed as follows: an intrahistiocytic location; a "halo" effect around each organism; and haematoxylinophilic nuclei stained with haematoxylin and eosin (H\&E). Periodic acid-Schiff (PAS) and Ziehl Neelsen (ZN) stains were negative. Based on the size of these yeasts, we classified them as H. capsulatum var capsulatum. Occasional Langhans' multinucleated giant cells were also visualised. There was fibrous thickening of the lymph node capsule as shown in figure 2 .

The kidneys showed marked corticomedullary differentiation; prominent whitish band between the cortex and the medulla. Histologically, there were mesangial sclerosis, cellular proliferation and thickened glomerular capsules. These were in keeping with membranoproliferative glomerulonephritis as indicated in figure 3 .

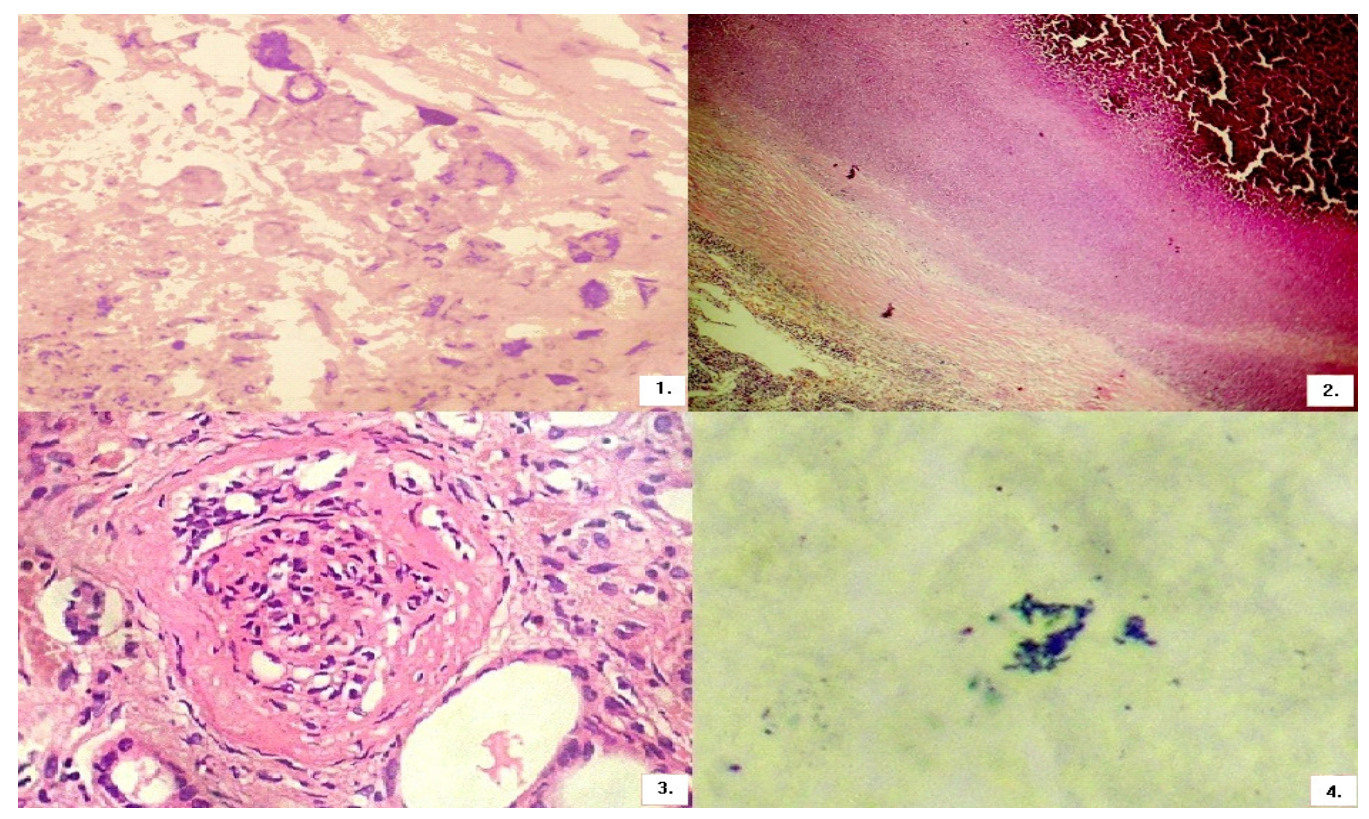

Figure 1: PAS staining technique showing viable yeast cells suggestive of histoplasmosis.

Figure 2: ZN staining is negative for tuberculosis.

Figure 3: Mesangial sclerosis, cellular proliferation and thickened glomerular capsules in keeping with membrano-proliferative glomerulonephritis

Figure 4: Grocots methenamine staining showing the typical morphological features of Histoplasma 


\section{Discussion}

We report a case of disseminated histoplasmosis in a 13-year-old girl. While this patient was alive, we did not consider histoplasmosis as a diagnosis. In a review of reported cases of disseminated histoplasmosis from South-East Asia, Wang et al $^{10}$ noted that none of the reports initially considered disseminated histoplasmosis as a possible diagnosis. Considerations were usually either tuberculosis or malignancies, and this was also the case in our patient until the post mortem. We considered tuberculosis and malignancies such as lymphomas but our investigations showed negative results. Therefore, it is important that clinicians managing children in areas where histoplasmosis has been reported are mindful of this possibility. There have been few reported cases of disseminated histoplasmosis in Nigeria. Seriki et al ${ }^{11}$ about three decades ago reported cases of disseminated histoplamosis involving two Nigerian children. The main clinical features noted in these children were fever, weight loss, lassitude lymphadenopathy, hepatosplenomegaly and severe anaemia which were similar to our index patient. They concluded that recognition of disseminated histoplasmosis in Nigeria is possible if it is considered in the differential diagnosis of pyrexia of undetermined origin and appropriate laboratory tests carried out on suitable specimens ${ }^{11}$.

During the examination of autopsy tissues, Cryptococcosis was considered in the differential diagnosis but was easily ruled out by the Grocots methenamine staining negativity of the organisms and the typical morphological features of Histoplasma that were observed (figure 4). Furthermore, extensive calcification of the lesions is another morphological indicator of histoplasmosis. Tuberculosis was also ruled out by the absence of acid fast bacilli in ZN staining. A Mantoux test carried out on the patient on presentation was also negative. The lung parenchyma did not show any caseation or signs of chronic lung disease and other organs of the body did not show features of miliary tuberculosis. A neutrophilic reaction at the edges of caseation in the lymph nodes suggests the diagnosis of a fungal disease such as histoplasmosis rather than tuberculosis. The most common sites of involvement for histoplasmosis, after the lung, are the reticuloendothelial tissues of the spleen, liver, lymph nodes and bone marrow. However, lesions have been documented in almost every organ and our patient also had kidney involvement.
Renal diseases associated with histoplasmosis can occur following direct infection of the kidney such as in recipients of renal transplants and this has led to allograft rejection ${ }^{12}$. It can also be immune complex mediated resulting in glomerulonephritis but it is rare ${ }^{13}$. Bullock et al in 1979 reported a transient mesangiopathic glomerulonephritis in a patient with disseminated histoplasmosis and eosinophilia ${ }^{14}$. In another case report in 1997, Burke et al noted a nephrotic range proteinuria in a 46 year old HIV positive black $\operatorname{man}^{13}$ who also had disseminated histoplasmosis. Renal biopsy in their patient demonstrated mesangio-proliferative glomerulonephritis. Our patient had membranoproliferative type of glomerulonephritis. While the patient was alive, she had proteinuria (not in nephrotic range), hematuria, hypertension and oedema in keeping with glomerulonephritis but also had renal insufficeincy. Proliferative glomerulonephritis with or without renal insufficiency has been associated with immune complex deposition ${ }^{13}$.

\section{Conclusion}

Disseminated histoplasmosis should be considered as a differential diagnosis of childhood chronic infections and malignancies as seen in Nigeria.

\section{Consent}

Written informed consent was obtained from the Care-giver of this patient.

\section{References}

1. Gutierrez ME, Canton A, Sosa N, Puga E, Talavera L. Disseminated Histoplasmosis in Patients with AIDS in Panama: A Review of 104 Cases. Clin Infect Dis. 2005; 40: 1199-1202.

2. Guimarães AJ, Nosanchuk JD, Zancopé-Oliveira, RM. Diagnosis of Histoplasmosis. Braz J Microbiol 2006; 37: 1-13.

3. Goodwin RA, Loyd JE, Des Prez RM: Histoplasmosis in normal hosts. Medicine 1981; 60: 231-66

4. Tobon AM, Franco L, Espinal D, Gomez I, Arango M, Trujillo H, Restrepo A: Disseminated histoplasmosis in children: the role of itraconazole therapy. Pediatr Infect Dis J 1996; 15: 1002-8.

5. Goodwin RA, Shapiro JL, Thurman GH, Thurman SS, Des Prez RM: Disseminated histoplasmosis: clinical and pathological correlations. Medicine 1980; 59:1-33.

6. Kauffman CA. Histoplasmosis: A clinical and laboratory update. Clin Microbiol Rev. 2007; 20: 115-32. 
7. Johnson PC, Khardori N, Najjar AF, Butt F, Mansell PW, Sarosi GA: Progressive disseminated histoplasmosis in patients with acquired immunodeficiency syndrome. Am J Med 1988; 85:152-8.

8. Johnson PC, Hamill RJ, Sarosi GA. Clinical review: progressive disseminated histoplasmosis in the AIDS patient. Semin Respir Infect 1989; 4 :139-46.

9. Martin RA, Williams T, Montalto N. AIDS with disseminated histoplasmosis. J Fam Pract 1989; 29:628-3.

10. Wang TL, Cheah JS and Holmberg K. Case report and review of disseminated histoplasmosis in South-East Asia: clinical and epidemiological implications. Trop Med Int Health 1996; 1: 35-42.

11. Seriki O, Aderele WI, Johnson A, Smith JA. Disseminated histoplasmosis due to histoplasma capsulatum in two Nigerian children.J Trop Med Hyg. 1975 Dec; 78(12):248-55.

12. Pourfarziani V, Taheri S. Is pulmonary histoplasmosis a risk factor for acute renal failure in renal transplant recipients? Saudi J Kidney Dis Transpl 2009; 20: 643-5

13. Burke DG, Emancipator SN, Smith, MC and Salata RA. Histoplasmosis and Kidney Disease in Patients with AIDS. Clin Infect Dis. 1997; 25 (2): 281-284.

14. Bullock WE, Artz, RP, Bhathena D, Tung, KS. Histoplasmosis: Association with Circulating Immune Complexes, Eosinophilia, and Mesangiopathic Glomerulonephritis. Arch Intern Med. 1979; 139 (6):700-702 